\title{
Extended Operational Range of Dual-Active-Bridge Converters by using Variable Magnetic Devices
}

\author{
Sarah Saeed, Jorge Garcia. \\ Dept. of Elec., Computer, and System Engineering \\ University of Oviedo \\ Gijón, Spain \\ e-mail: hazkialsarah.uo@uniovi.es, garciajorge@uniovi.es.
}

\begin{abstract}
This paper explores the use of a variable inductor as an energy transfer reactive element in a Dual-Active-Bridge (DAB) converter. By using a controlled variable inductor, the optimal switching region in the operation of the phase-shift DAB can be extended, and thus high efficiencies can be achieved over wide-load ranges. Moreover, the combination of the variable inductance with the phase shift as two independent control parameters allows for the linearization of the DAB converter transfer function, which gives significant merit to the implementation of the control function. And finally, it is feasible to reduce the magnetic core size, due to the controlled saturation of the device. To study those proposed improvements, a circuitbased model is developed to simulate the converter system. Furthermore, a prototype is constructed for the DAB converter including a controlled variable inductor and preliminary experimental results are presented which validate the studies carried out.
\end{abstract}

Keywords-dual-active-bridge, variable magnetics, hysteresis losses, efficiency.

\section{INTRODUCTION}

Variable magnetic elements allow the inclusion of additional degrees of freedom in the design and control of Power Electronic Converters (PECs). This is particularly useful in resonant converters, where the output power is generally controlled by varying the switching frequency due to the power vs. frequency characteristic of the converter. This standard frequency control presents some drawbacks due to Electro-Magnetic Interference (EMI) issues, complex filtering, or variable sampling time, among others, especially for a wide range of variation. However, if variable magnetics are used, the same control margins can be obtained at a constant switching frequency, therefore allowing for an optimization of the EMI filters and sampling procedures [1][2].

In other applications such as the Dual-Active-Bridge (DAB) converter, the addition of a new degree of freedom to the control scheme by means of the Variable Inductor (VI) can increase the operation parameters such as the soft switching margins, as reported in preliminary works [3] [4]. Specifically, in [3], the variable inductor concept has been proposed as the main energy transfer element for Dual-HalfBridge (DHB) converters, the study concluded that the ZeroVoltage-Switching (ZVS) operation region can be extended for the phase shift dual-bridge DC-DC converters by employing an adaptive inductor. Therefore, high efficiency can be achieved over wide-load range. On the other hand, for the DAB converter, the authors in [4] adopted inserting a further series inductor at light load conditions to decrease circulating currents and allow an increased efficiency.

This work has been partially supported by the government of the Principality of Asturias, under "Severo Ochoa" program of predoctoral grants for training in research and university teaching, grant number BP16133. Also, the work has been partially supported by the Spanish Government, under research grant ENE2016-77919, Project "Conciliator" (Innovation Development and Research Office-MEC).
Consequently, in the aspect of improving the latter mentioned converter's efficiency, these studies reinforce the idea of introducing an adaptive power transfer inductor in the DAB converter to reduce the overall power losses.

Additionally, this technique has potential features, such as linearization of the control to output transfer function, which also implies the extension of the maximum power level. Moreover, it is postulated that regarding the closed loop control of the converter under disturbance rejection, the adaptive inductor adds an extra control parameter and thus can be employed to assist the main control scheme in riding through the transient event. It is worth to notice that replacing the fixed power transfer inductance by a variable one can allow for core size optimization and achieving higher power density at the cost of additional power electronic devices needed for controlling the device saturation [5]. In all cases, it is necessary to investigate the overall PEC incorporating the variable magnetic device.

The aim of this work is to study the potential extension of the operation range of a DAB converter brought about by using a variable inductor. The analysis is carried out using an electromagnetic system simulation model which takes into consideration different component losses, such as switching and conduction losses, magnetic device core and winding losses, etc. In this context, section II states the structure and the principle of operation of the conventional DAB converter. Also, the latter section provides a brief about the variable inductor structure, operation and model. Section III opens several aspects for the potential improvements that can be achieved by using a variable inductor in a DAB converter. In section IV, the test setup and the experimental results are presented. And finally, the conclusions of the work are summarized in section V.

\section{PRoposed DAB USING VARIABLE INDUCTOR}

\section{A. Principle of operation of conventional DAB converter}

The DAB converter is a bidirectional isolated DC-DC converter which is composed of two full-bridge circuits connected across a constant power transfer inductor and a high frequency (HF) transformer, as clarified by Fig. 1.

The flexibility of the primary and secondary active full bridges in the DAB allows for various modulation methods to minimize the losses and to maximize the efficiency over a wide load range and wide operation voltage range [6][7]. Using the simplest modulation, Single Phase Shift (SPS), the full-bridge converters are operated at constant switching frequency, with maximum duty cycles, 0.5 , while the bridge leading by a phase shift, $\theta$, sends power to the lagging one [8]. 


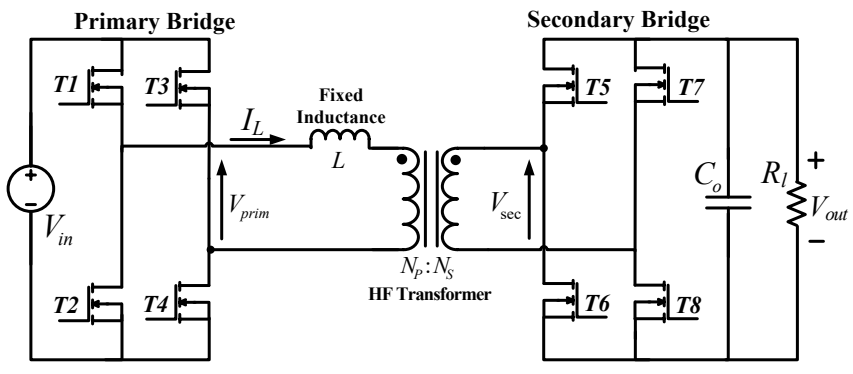

Fig. 1. DAB converter topology.

Equation (1) shows the analytical expression for the power flow in the converter.

$$
P_{D A B}=\frac{V_{\text {in }} \cdot V_{o u t}}{X_{L} \cdot r_{t}} \cdot \theta \cdot\left(1-\frac{\theta}{\pi}\right),
$$

As it can be observed from Eq. (1), the control parameters that affect the power transfer are the input and output voltage levels, $V_{\text {in }}$ and $V_{\text {out }}$ respectively, the transformer turns ratio, $r_{t}=N_{s} / N_{p}$, the phase shift between the primary and secondary bridges, $\theta$, and the impedance, $X_{L}=2 . \pi$.f.L where $f$ is the switching frequency and $L$ is the inductance. Therefore, replacing the fixed inductor by a variable one can serve as an extra control parameter for the power transfer in the converter.

\section{B. Variable inductor: structure, operation, and model}

From the study of the state of the art, the double E-core structure, depicted in Fig. 2, is selected to be the most appropriate and most comprehend in literature [9].

The current flowing through the main winding, $N_{c}$, generates an $\mathrm{AC}$ flux, $\phi_{C}$, which circulates through the center arm and splits to the outer right and left arms. Applying a relatively small DC current, $I_{b}$, to the bias control windings, $N_{b}$, a DC flux, $\phi_{R}$ or $\phi_{L}$, is produced which tends to circulate mainly through the outer (ungapped) circumference of the core [9]. This DC flux will bias the operation point of the magnetic material within the $\mathrm{BH}$ curve towards nonlinear region, causing a variation in the inductance seen from the main winding terminals.

The variable inductor is modeled based on the reluctance equivalent circuit. The circuit corresponding to the double-E core variable inductor is illustrated in Fig. 3. The magnetic core is modeled using three main elements: the constant reluctance, which models the non-ferromagnetic material, such as the air gap, $R_{\text {gap }}$. Secondly, the variable reluctance, which models the non-linear behavior of ferro-magnetic material, such as the left, right, and center arms, represented by $R_{L}, R_{R}$, and $R_{C}$ respectively. And finally, the magnetomotive source, which models the winding electrical and magnetic interaction, such as the control windings represented by $N_{b} . I_{b}$, and the main winding represented by $N_{c} . I_{c}$. The full developed model is described in detail in a previous publication by the authors [10]. The latter model takes into consideration device losses, mainly core losses and winding Eddy current losses. The following section clarifies the inductance variation as a function of the bias control current, and moreover, the effect of this variation on the power transfer in the proposed DAB converter.

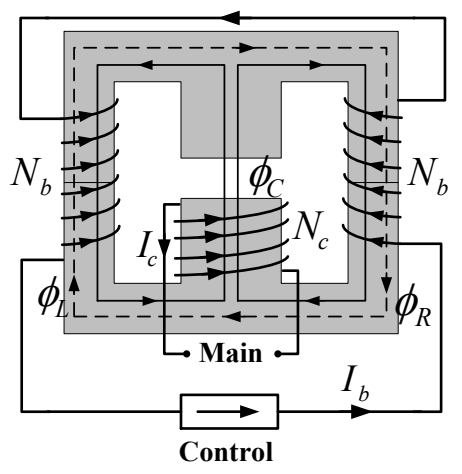

Fig. 2. Variable inductor based on double E-core structure.

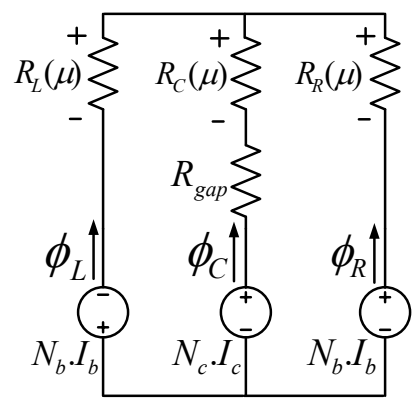

Fig. 3. Reluctance equivalent circuit of double E-core variable inductor shown in Fig. 2.

\section{ELECTROMAGNETIC SYSTEM SIMULATIONS}

This section is intended to demonstrate the simulation results of the DAB converter including the variable magnetic element. Emphasis is given to the improvements brought about by replacing the fixed inductor in a conventional DAB converter by a variable one.

\section{A. Control of power transfer using variable inductor}

As mentioned in the previous section, the inductance is varied by means of controlled saturation of the magnetic core using a DC bias control current. Fig. $4 \mathrm{a}$ depicts the inductance variation as a function of the bias control current, and Fig. $4 \mathrm{~b}$ shows the resulting variation in the power transfer in the DAB converter. This illustrates that the variable inductor can serve as a control parameter for the power transfer in the converter.

Fig. 5 further adds a third dimension to depict the effect of phase shift as a control parameter in combination with the inductance variation. As it can be observed, at a certain inductance value, as the phase shift increases the power transfer in the DAB converter increases correspondingly. However, this increase of the power becomes less significant as the inductance value increases. Designing the inductor for a specific operation range is, hence, a critical process in a DAB converter.

On the other hand, Fig. 6 defines the limitations imposed by the phase shift control parameter on the variable inductance one. The figure clarifies that for lower phase shift smaller amount of bias current in the inductor control windings can achieve a comparably large output power. In conclusion, the effect of bias current is decreased as the phase shift between bridges increases towards maximum. 


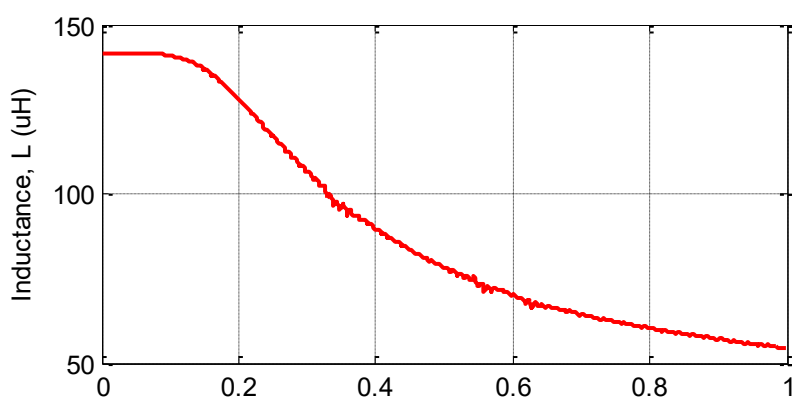

(a)

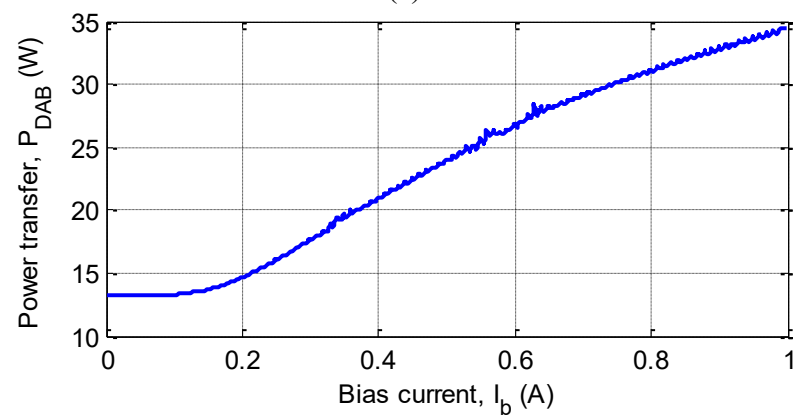

(b)

Fig. 4. Effect of inductance variation on power transfer in the proposed DAB converter, (a) inductance as a function of bias current, (b) RMS power transfer in DAB converter as a function of bias current.

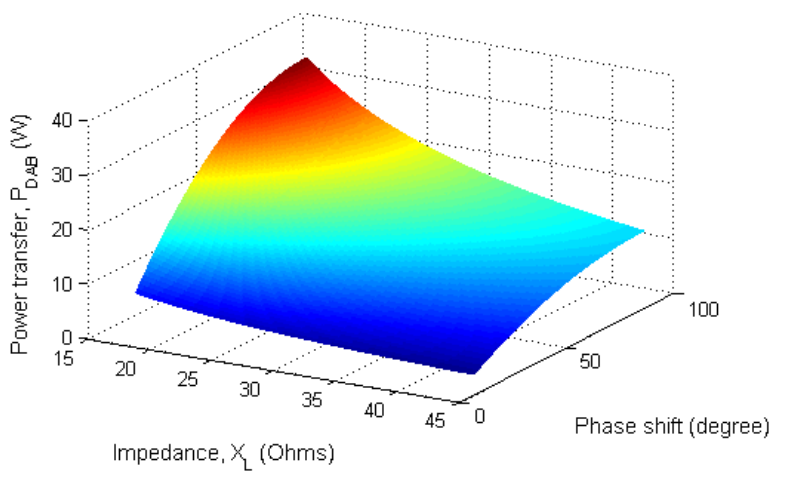

Fig. 5. Power transfer in a $\mathrm{DAB}$ converter as a function of inductance and phase shift.

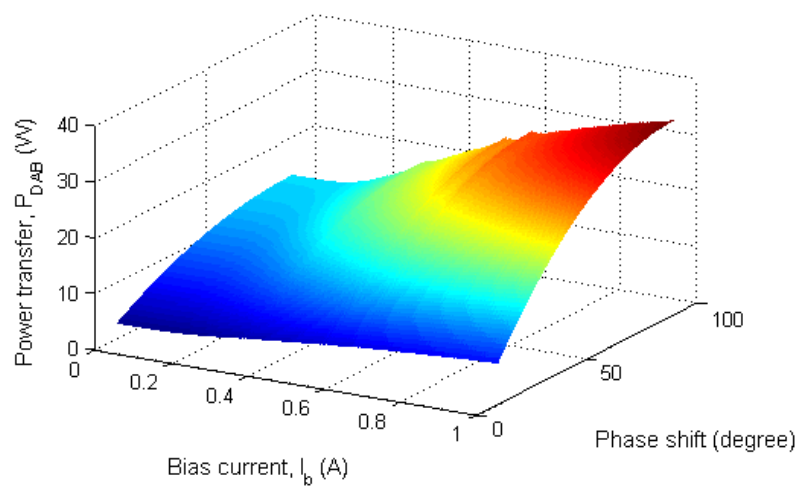

Fig. 6. Power transfer in a DAB converter as a function of bias current and phase shift.

\section{B. Linearization of the control function}

As explained in the previous section the power transfer of a DAB converter is regulated by Eq. (1) and is a function of several parameters. Fig. 7a shows the power transfer in the $\mathrm{DAB}$ converter as a function of the phase shift. It can be observed that the power transfer increases linearly with the increase of phase shift until a certain limit after which the rate of increase of power drops following a sinusoidal waveform until reaching the maximum power transfer at 90 degrees phase-shift. This characteristic operation is intrinsic to the SPS DAB converter, and thus to control the power transfer in the converter, the phase shift is used as the primary control parameter. By manipulating Eq. (1), one can reach to Eq. (2) which explains the phase shift as a function of required output power.

$$
\theta=\frac{\pi}{2} \cdot\left(1 \pm \sqrt{1-\frac{4 \cdot P_{D A B} \cdot X_{L} \cdot r_{t}}{\pi \cdot V_{\text {in }} \cdot V_{\text {out }}}}\right)
$$

As is can be observed the equation is quite non-linear introducing difficulty in implementation and excessive processing time. Therefore, it is promising to use the inductance as an extra control parameter to allow the linearization of the power transfer function, and thus achieving a linear control function. Fig. 7a illustrates the nonlinear power transfer curve of the conventional DAB converter, compared to the linearized one achieved by making use of the variation in the inductance. The inductance is constant during the linear region as demonstrated in Fig. $7 \mathrm{~b}$, while in order to increase the power transfer and keep the linearity of the function, the inductance is reduced by means of varying the bias current command as shown in Fig. 7c.

\section{Efficiency improvement}

At light loading condition, i.e. $V_{\text {out }} \ll r_{t} . V_{\text {in }}$, two issues take place which contribute significantly to the reduced efficiency in this operation region. First, the loss of ZVS due to insufficient energy required to charge and discharge the MOSFET capacitors causing elevated switching losses [3]. And secondly, relatively small phase shift values interfere with the deadtime range causing a phenomenon called "voltage polarity reversal" [11] [12] and limiting the minimum power transfer. Those two issues cause an increase in the reactive power component and consequently reduce the efficiency at this load range. Moreover, at high loading condition, i.e. $V_{\text {out }} \gg r_{t} . V_{\text {in }}$, relatively large circulating currents take place causing an increase in conduction losses and elevate reactive power losses [13] [14].

In this context, varying the inductance to have a relatively higher value at light load allows for increasing the phase shift and maintaining ZVS for a wider range, as well as avoiding the interference with the deadtime. And on the other hand, decreasing the inductance value at heavy load allows for reducing the circulating currents. This is illustrated by Fig. 8a., which compares the inductance in a conventional DAB converter to that of the proposed DAB using variable inductor. The resulting phase shift is shown in Fig. 8b, and finally, the improvement in efficiency can be clearly observed in Fig. 8c. 


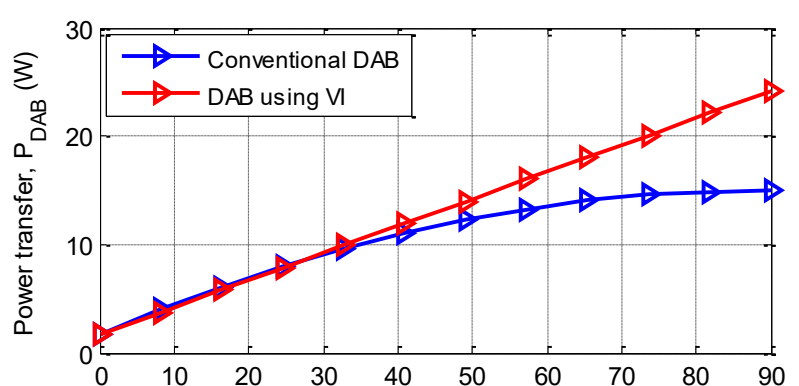

(a)

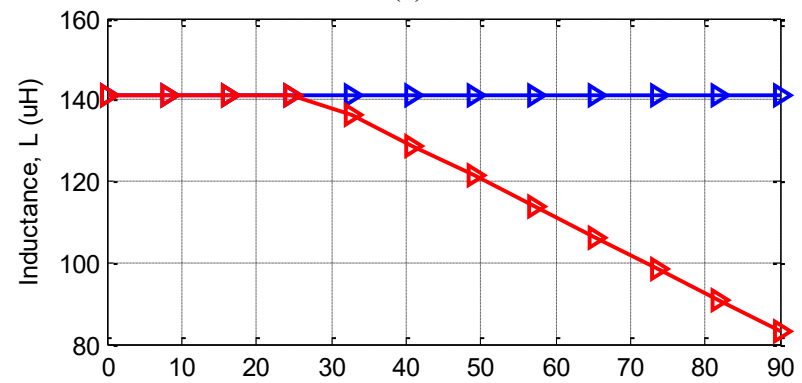

(b)



(c)

Fig. 7. Linearization of power transfer characteristics in the proposed DAB converter using VI, (a) output power transfer, (b) inductance, and (c) bias control current command as a function of phase shift angle.

\section{EXPERIMENTAL RESULTS}

\section{A. Experimental platform}

A test platform has been developed to validate the electromagnetic model and the system analysis. The platform consists of a power stage and a programmer stage. The power stage includes the DAB converter as well as the variable inductor. To supply the control winding of the variable inductor a $\mathrm{DC}$ voltage source is used in series with a resistor to have a maximum of $1 \mathrm{~A}$ DC bias control current.

On the other hand, the programmer stage consists of a microcontroller peripheral board as well as a software platform for providing the switch modulation and processing the measurements. The detailed specifications for the whole test setup are provided in Table I, and Fig. 9 illustrates a schematic of the hardware connections. Moreover, Fig. 10 shows a picture of the constructed experimental platform.

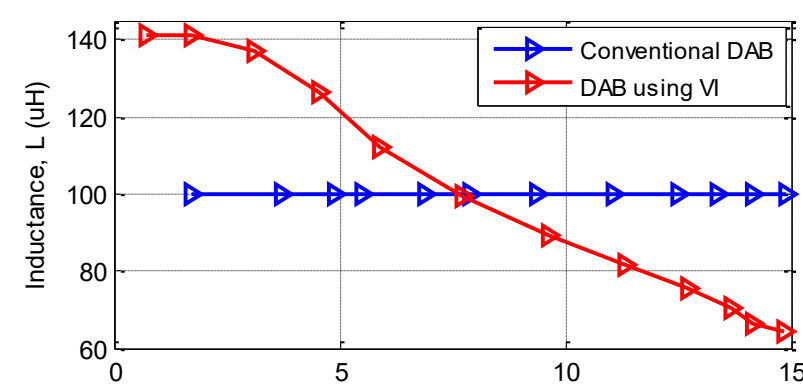

(a)

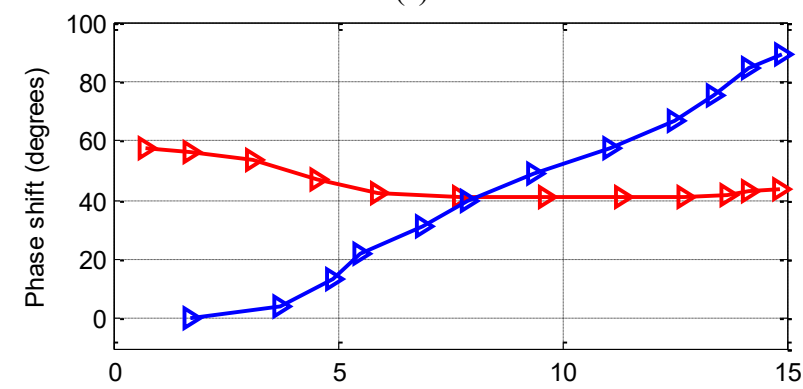

(b)

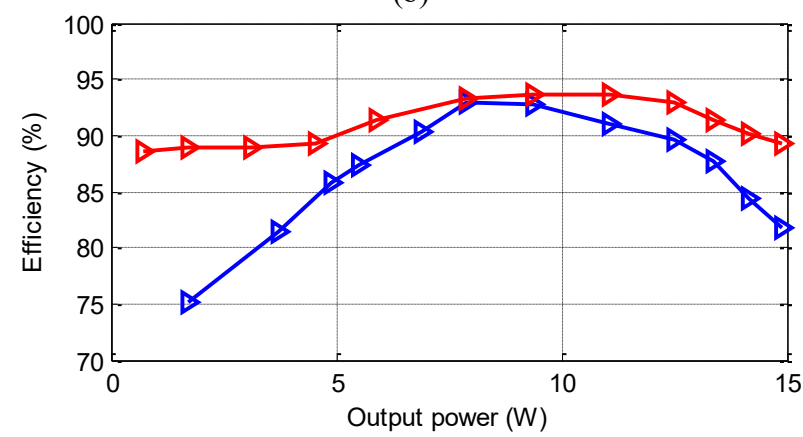

(c)

Fig. 8. Efficiency improvement of DAB converter using variable inductor, (a) inductance value, (b) phase shift, and (c) converter efficiency as a function of output power transfer.

TABLE I. Specifications of the Test Setup

\begin{tabular}{|c|c|c|}
\hline \multicolumn{3}{|c|}{ Power Stage } \\
\hline \multirow{3}{*}{$\begin{array}{l}\text { Variable } \\
\text { Inductor }\end{array}$} & Magnetic core & $\begin{array}{c}\mathrm{N} 87 \\
\text { ETD49/25/16 }\end{array}$ \\
\hline & Main winding no. of Turns $\left(N_{c}\right)$ & 23 turns \\
\hline & Bias winding No. of Turns $\left(N_{b}\right)$ & 55 turns \\
\hline \multirow{7}{*}{$\begin{array}{c}D A B \\
\text { Converter }\end{array}$} & Input voltage & $25 \mathrm{~V}$ \\
\hline & Output voltage & $30 \mathrm{~V}$ \\
\hline & Maximum current & $3 \mathrm{~A}$ \\
\hline & Frequency & $50 \mathrm{kHz}$ \\
\hline & Deadtime & $2 \mathrm{us}$ \\
\hline & Transformer and turns ratio & $\begin{array}{c}\text { Himag planar } \\
\text { transformer 18:22 }\end{array}$ \\
\hline & Switches & $\begin{array}{l}\text { SiC MOSFETs } \\
\text { SCT2280KE }\end{array}$ \\
\hline \multicolumn{3}{|c|}{ Programmer Stage } \\
\hline Hardware & Microcontroller & $\begin{array}{l}\text { Texas Instruments } \\
\text { TMS320F28335 }\end{array}$ \\
\hline Software & Platform & \begin{tabular}{|c|} 
Code Composer \\
Studio 6.1 .1
\end{tabular} \\
\hline
\end{tabular}




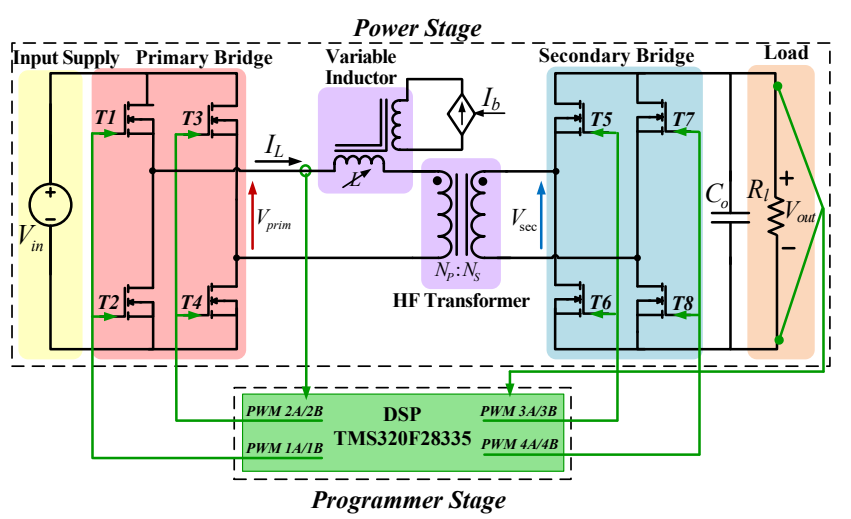

Fig. 9. Schematic of experimental setup.

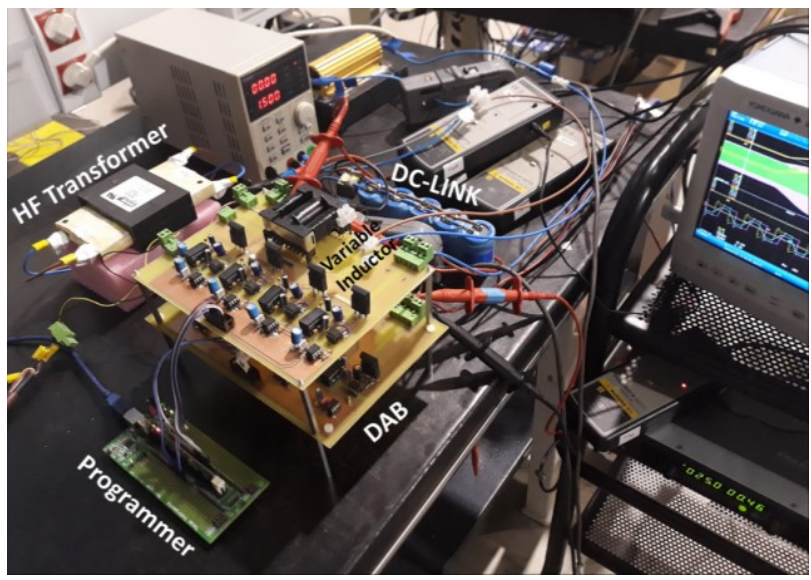

Fig. 10. Experimental setup.

\section{B. Inductance effect on power transfer in DAB converter}

To test the effect of inductance variation on the DAB power transfer, the phase shift is set to the maximum, 90 degrees, and the bias control current has been varied from 0 to $1.5 \mathrm{~A}$. The inductance is computed from the measured voltage across the variable inductor main winding, $V_{L}$, and the current flowing through it, $I_{L}$, and the inductance waveform as a function of bias control current is illustrated in Fig. 11a. It can be observed that as the bias current increases, the inductance decreases, thus the inductor current increases correspondingly, and consequently the power transfer, as shown in Fig. 11b. The voltage and current waveforms of the variable inductor are shown in Fig. $11 \mathrm{c}$ and Fig. $11 \mathrm{~d}$, for $0 \mathrm{~A}$ bias current and $1.5 \mathrm{~A}$ bias current, respectively.

\section{CONCLUSIONS}

A DAB converter using variable inductor has been proposed. An electromagnetic model has been developed which describes the behavior of a variable inductor using the reluctance equivalent circuit and including the device losses, also the variable inductor was simulated within a DAB converter in a circuit-based time domain simulation. Using these models, a new degree of freedom was explored for the control of power transfer in the $\mathrm{DAB}$ converter using variable inductor, as well as studying the margins for combining both phase shift control with the variable inductance one.

Moreover, the analysis opened several aspects for the improvement of $\mathrm{DAB}$ converter operation using the variable inductor solution, such as extending the limits of the power transfer in the converter, linearization of the control function, and boosting the converter efficiency over wider load range. The overall efficiency of the proposed DAB exceeds the conventional converter by around $10 \%$ at light and heavy loading conditions.

The preliminary experimental results clarified the effect of the inductance variation on the power transfer in a DAB converter under maximum phase shift.

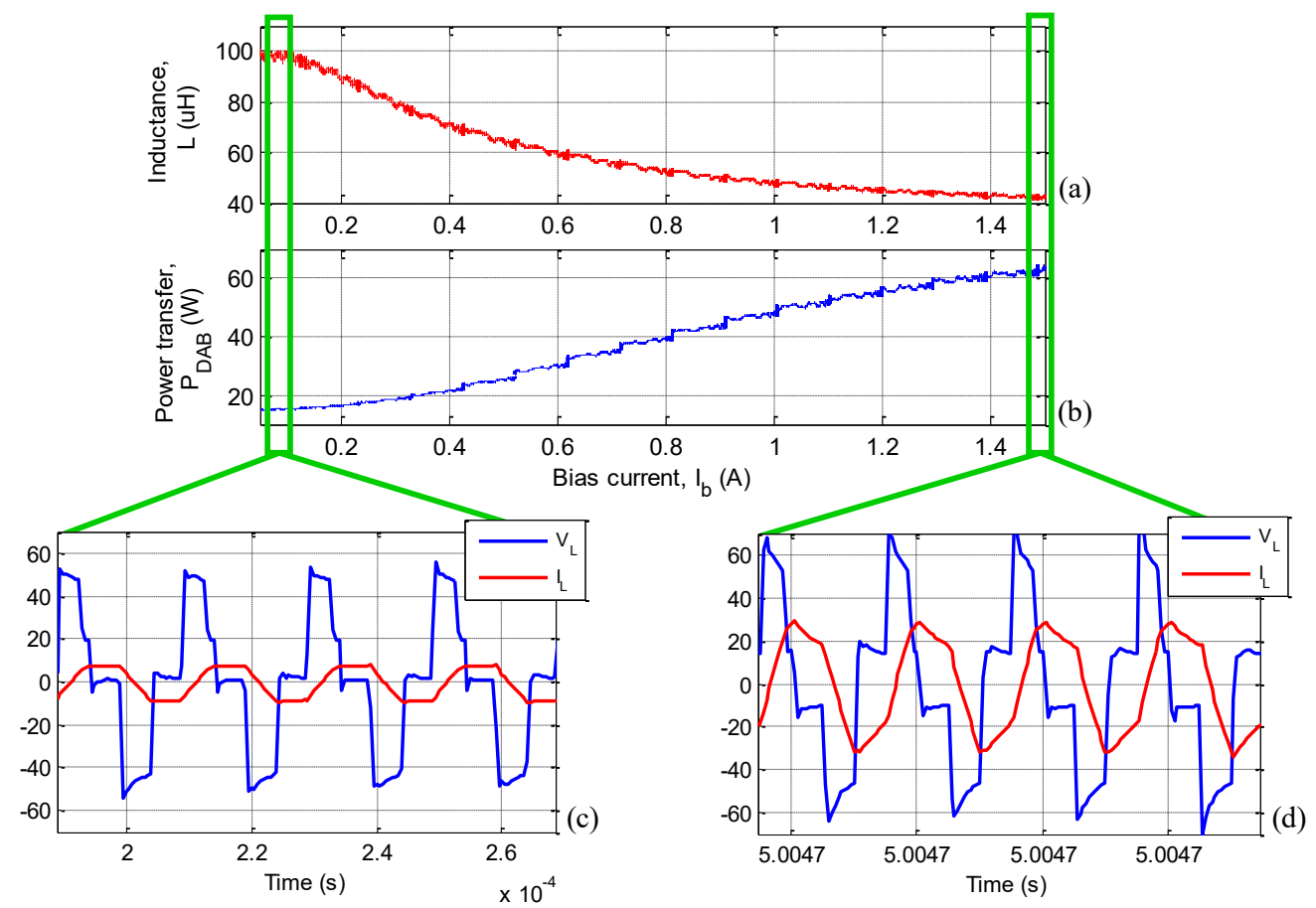

Fig. 11. Experimental results - (a) variation of inductance, (b) corresponding varaition of power transfer in DAB converter, (c) inductor voltage and current (scaled by 10 ) at $0 \mathrm{~A}$ bias current, and (d) inductor voltage and current at $1.5 \mathrm{~A}$ bias current. 


\section{REFERENCES}

[1] M. S. Perdigão, M. Menke, A. R. Seidel, R. A. Pinto and J. M. Alonso, "A review on variable inductors and variable transformers: Applications to lighting drivers," 2014 IEEE Industry Application Society Annual Meeting, Vancouver, BC, 2014, pp. 1-8.

[2] M. S. Perdigão, B. Baptista, H. Marques, E. S. Saraiva and J. M. Alonso, "On the use of magnetic regulators in electronic ballasts," 45th International Universities Power Engineering Conference UPEC2010, Cardiff, Wales, 2010, pp. 1-6.

[3] H. Fan and H. Li, "High-Frequency Transformer Isolated Bidirectional DC-DC Converter Modules with High Efficiency Over Wide Load Range for $20 \mathrm{kVA}$ Solid-State Transformer," in IEEE Transactions on Power Electronics, vol. 26, no. 12, pp. 3599-3608, Dec. 2011.

[4] Burgio, A.; Menniti, D.; Motta, M.; Pinnarelli, A.; Sorrentino, N.; Vizza, P., "A laboratory model of a dual active bridge DC-DC converter for a smart user network," in Environment and Electrical Engineering (EEEIC), 2015 IEEE 15th International Conference on, vol., no., pp.997-1002, 10-13 June 2015.

[5] Marina S. Perdigão, João Pedro F. Trovão, J. M. Alonso, and E. S. Saraiva, "Large-Signal Characterization of Power Inductors in EV Bidirectional DC-DC Converters Focused on Core Size Optimization," IEEE Transactions on Industrial Electronics, Vol. 62, No. 5, May 2015.

[6] F. Krismer and J. W. Kolar, "Efficiency-Optimized High-Current Dual Active Bridge Converter for Automotive Applications," in IEEE Transactions on Industrial Electronics, vol. 59, no. 7, pp. 2745-2760, July 2012.

[7] F. Krismer, S. Round and J. W. Kolar, "Performance optimization of a high current dual active bridge with a wide operating voltage range," 2006 37th IEEE Power Electronics Specialists Conference, Jeju, 2006, pp. 1-7.

[8] A. Rodríguez, J. Sebastian, D. G. Lamar, M. M. Hernando, and A. Vazquez, "An overall study of a dual active bridge for bidirectional DC/DC conversion," in Proc. IEEE Energy Convers. Congr. Expo., Sep.2010, pp. 1129-1135.

[9] D. Medini and S. Ben-Yaakov, "A current-controlled variable inductor for high frequency resonant power circuits," in Proc. IEEE APEC, 1994, pp. 219-225.

[10] S. Saeed, J. García, R. Georgious, "Modeling of variable magnetic elements including hysteresis and Eddy current losses," 2018 IEEE Applied Power Electronics Conference and Exposition (APEC), San Antonio, TX, 2018, pp.1750-1755.

[11] Y. Xie, J. Sun, and J. S. Freudenberg, "Power flow characterization ofa bidirectional galvanically isolated high-power dc-dc converter over awide operating range," IEEE Trans. Power Electron., vol. 25, no. 1,pp. 54-66, Jan. 2010.

[12] B. Zhao, Q. Song, W. Liu, and Y. Sun, "Dead-time effect of the high frequency isolated bidirectional full-bridge $\mathrm{dc}-\mathrm{dc}$ converter: Comprehensive theoretical analysis and experimental verification," IEEE Trans. Power Electron., vol. 29, no. 4, pp. 1667-1680, Apr. 2014.

[13] H. Bai and C. Mi, "Eliminate reactive power and increase system efficiency of isolated bidirectional dual-active-bridge DC-DC converters using novel dual-phase-shift control," IEEE Trans. Power Electron., vol. 23,no. 6, pp. 2905-2914, Nov. 2008.

[14] Sarah Saeed, "Design and construction of an isolated DC to DC switching converter for integration of energy storage systems in power electronic applications," M.S. thesis, Dept. of Elec. Eng., Univ. of Oviedo, Spain, 2015. Accessed on: Jul. 23, 2015. [Online]. Available: http://hdl.handle.net/10651/33257 